\title{
Dynamic characteristics of light emission accompanying cryocondensation of nitrous oxide and ethanol
}

\author{
A. Drobyshev, A. Aldiyarov, and E. Korshikov \\ Al-Farabi Kazakh National University, Almaty 050038, Kazakhstan \\ E-mail: Andrei.drobyshev@kaznu.kz
}

Y.M. Strzhemechny

Texas Christian University, Texas, US

Received January 8, 2015, revised February 2, published online May 25, 2015

\begin{abstract}
We report new results in the study of cryoradiation accompanying the condensation of some gas. The main objectives of this research are: (i) check a broader range of substances for their ability to cryoradiation; (ii) the study of the nature of radiation and its relaxation characteristics; (iii) the study of thermally stimulated processes in condensed samples. Of the compounds tested only nitrous oxide and ethanol exhibited the ability to generate cryoradiation during condensation from the gas phase. Separate flashes detected by a photomultiplier represent a superposition of a series of successive relaxation processes of individual regions, somehow related to each other. It is possible that such areas are crystallites containing a set of molecules of nitrous oxide/ethanol, which are in metastable states with different activation energies. Radiation by these molecules during the transition into the ground state induces successive relaxation processes of molecular groups according to their activation energy distributions. Nonequilibrium radiative states forming in the process of cryocondensation of nitrous oxide and ethanol may have different nature of the activation energy distribution and this could explain the difference in the character of the radiation relaxation in these substances.
\end{abstract}

PACS: 61.50.-f Crystal structure;

78.30-j Infrared and Raman spectra;

68.35.Rn Phase transitions and critical phenomena.

Keywords: cryocondensate, radiation, ethanol, nitrous oxide, relaxation

\section{Introduction}

Detection and subsequent studies of light emission in a cryocondensate of nitrous oxide reported by us [1-3] have been stimulating further efforts for detailed elucidation of the physical and chemical nature of this phenomenon. This yielded, in the past few years, a series of publications on the subject by other groups, such as, e.g., [4], where the authors discovered the effect of spontaneous dipole ordering in thin films of a cryocondensate of nitrous oxide accompanied by generation of an electric potential between the substrate and the interfacial surface. Further reports by this group [5-7] identified a number of other substances (propane, nitrous oxide, methyl formate) exhibiting similar remarkable properties. In the most recent articles by Field et al. [5,8,9], a model has been suggested to explain these experimental results. This model is "put forward based upon competition between dipole alignment and thermal disorder, which is successful in reproducing the variation of the degree of dipole alignment and the spontaneous electric field with deposition temperature" [5].

Another area attracting in recent years researchers working to address peculiar properties of nitrous oxide has been the theoretical and experimental exploration of isomorphic states in the $\mathrm{N}_{2} \mathrm{O}$ molecule [10-12]. These forms of the nitrous oxide molecule have different makeup of the valence bonds as well as the outer shell electronic configurations and, thus, different magnitudes of the dipole moment. This is not only a consequence of the interaction between the nitrous oxide molecule and the surface $[10,11]$, but may have a more complex nature $[12,13]$.

Calculations carried out in [13] demonstrate that in addition to the main linear $\mathrm{N}-\mathrm{N}-\mathrm{O}$ state of the molecule there are two local energy minima - a relatively stable cyclic isomer with the ground state energy of $2.81 \mathrm{eV}$ and an unstable linear $\mathrm{N}-\mathrm{O}-\mathrm{N}$ isomer with the energy of $4.80 \mathrm{eV}$.

In our work [1] we have also discovered an interesting behavior of nitrous oxide during both cryocondensation 
and a subsequent thermal cycling of the condensed sample [2]. Thus, studies of the dynamic characteristics of the gassolid phase transitions indicated that the process of cryocondensation of nitrous oxide on to a metal substrate is accompanied by visible light generation [1-3]. Spectral analysis of the cryoradiation revealed presence of two emission bands at the wavelengths of $380 \mathrm{~nm}$ and $700 \mathrm{~nm}$ [2]. In $[3,14]$ we proposed an explanation of the observed phenomenon employing ideas formulated in [4,5,13], which, we believe, demonstrate a robust consistency between our results and those reported by other groups.

In this paper we report on our new results for the radiation that accompanies cryocondensation of certain gases. The main objectives of this research were to test a wider range of substances for their ability to produce the cryocondensation radiation, to study the dynamics and the relaxation of this radiation, and to analyze the thermally stimulated processes in the condensed samples.

\section{Experimental}

The studies were conducted at the facility and according to the procedure described previously by us in detail $[2,3,14]$, thereby here we will outline only the basic aspects of the experiment. The main unit of the setup is a cylindrical vacuum chamber with a diameter and a height of $450 \mathrm{~mm}$. The evacuation of the chamber was carried out by a turbomolecular pump Turbo-V-301.The ultimate vacuum in the chamber was $10^{-8}$ Torr or better. The conden- sation pressure was set in the range $P=\left(10^{-4}-10^{-2}\right)$ Torr. Measurement of the pressure in the chamber was carried out by a wide-range pressure gauge FRG-700 with an AGC-100 controller. A Gifford-McMahon microcryogenic system is installed in the center of the chamber. A mirror substrate serving as the condensation surface is mounted at the top flange of the microcryogenic system. The substrate, $60 \mathrm{~mm}$ in diameter, is made of copper, with the working surface coated by silver. The minimum condensation temperature is $12 \mathrm{~K}$. The temperature was measured with a DT 670-1.4 silicon sensor coupled with a M335/20s temperature controller. Photomultiplier tubes P25a-SS-0-100 with a sampling frequency of $10^{5} \mathrm{~s}^{-1}$ were employed to measure the light emission intensity.

\section{Results and discussion}

When choosing substances to study we have considered the possible role of the dipole moment of the molecules as one of the factors responsible for the cryoradiation. Thus, for comparison purposes, we investigated both the dipole molecules (nitrous oxide, water, ethanol) and molecules with a zero intrinsic dipole moment (nitrogen, carbon dioxide, argon). Condensation of these gases was carried out at a pressure of the gas phase of $P=10^{-2}$ Torr onto a substrate at $T=16 \mathrm{~K}$. In our studies it was found that among the chosen gases only ethanol, along with nitrous oxide, exhibited the ability to emit light during condensation. Condensation of $\mathrm{Ar}, \mathrm{N}_{2}, \mathrm{CO}_{2}$ and $\mathrm{H}_{2} \mathrm{O}$ under the same conditions occurs
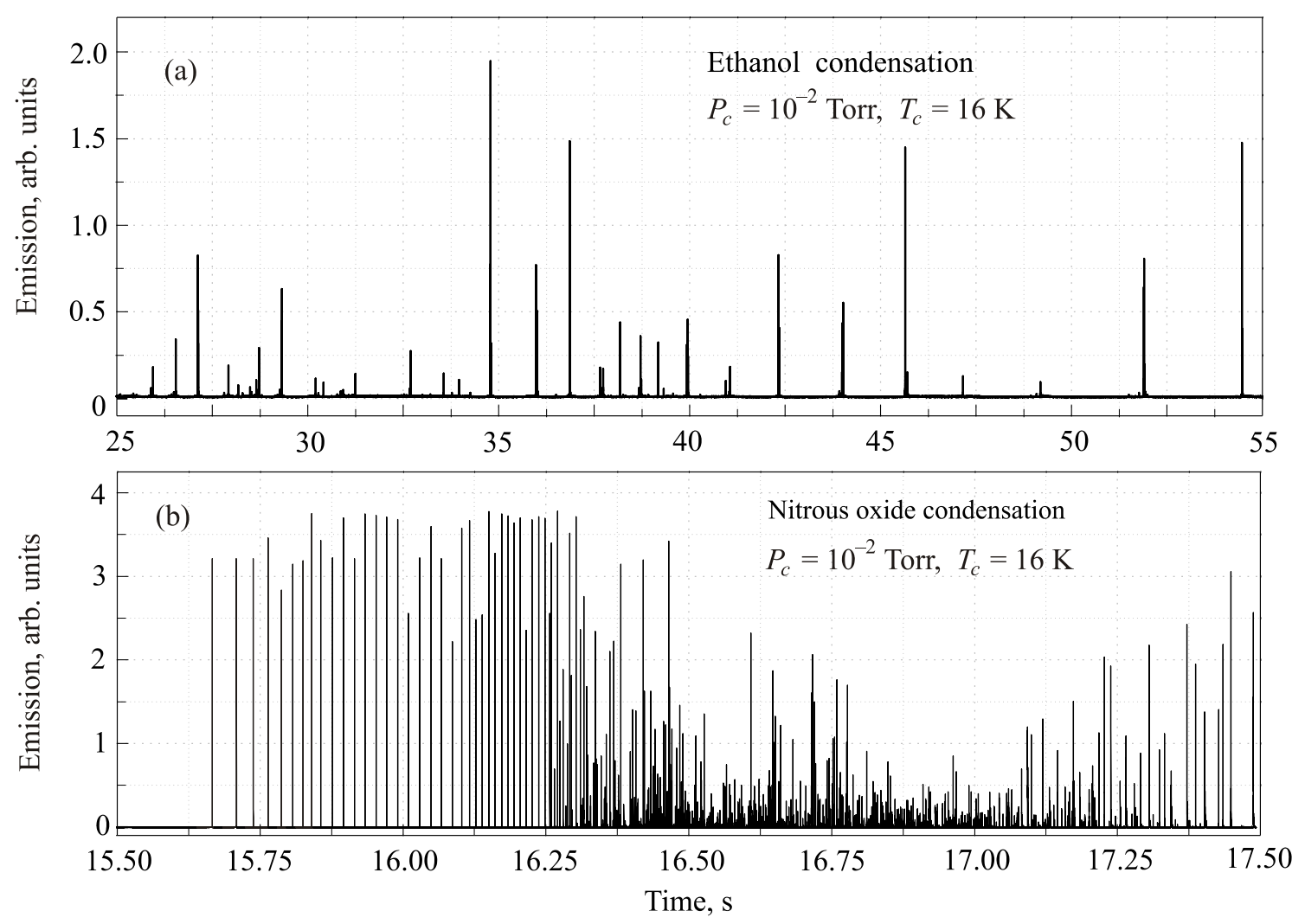

Fig. 1. Cryocondensation radiation detection in $\mathrm{N}_{2} \mathrm{O}$ and $\mathrm{C}_{2} \mathrm{H}_{5} \mathrm{OH} P_{c}=10^{-2}$ Torr, $T_{c}=16 \mathrm{~K}$ 
without light radiation. This fact, on the one hand, may be indicative of an improper assumption about the role of the intrinsic dipole moments as a crucial factor in the nature of the observed phenomenon. On the other hand, the absence of radiation during condensation may be an indirect confirmation of the fact that the condensation radiation observed for $\mathrm{N}_{2} \mathrm{O}$ and $\mathrm{C}_{2} \mathrm{H}_{5} \mathrm{OH}$ is not a triboluminescence or the result of cracking of the samples. Otherwise we would have detected radiation in the process of cryocondensation water, argon, carbon dioxide and nitrogen.

Our data demonstrating that not only nitrogen monoxide but also ethanol has the ability to yield the cryocondensation radiation are presented in Fig. 1. The $x$ axes denote the condensation time while the $y$ axes - the photomultiplier signal readout during cryocondensation of ethanol (a) and nitrous oxide (b).

As one can see in Fig. 1, during condensation under the same conditions onto the substrate at $T=16 \mathrm{~K}$, the $\mathrm{N}_{2} \mathrm{O}$ and $\mathrm{C}_{2} \mathrm{H}_{5} \mathrm{OH}$ molecules produce radiation with a very different time density of the radiation bursts, which is significantly higher for nitrous oxide than for. ethanol. Furthermore, from our data for both $\mathrm{N}_{2} \mathrm{O}$ and $\mathrm{C}_{2} \mathrm{H}_{5} \mathrm{OH}$ it follows that there is a threshold value of the condensation pressure, below which there is no radiation, and this minimum pressure is different for the two studied compounds. Thus, for nitrous oxide the cryocondensation emission onset is at $P$ $10^{-5}$ Torr, whereas for $\mathrm{C}_{2} \mathrm{H}_{5} \mathrm{OH}$ the corresponding value is $P=10^{-2}$ Torr. It should be pointed out that for both substances the cryocondensation radiation is visible with a naked eye.

For $\mathrm{C}_{2} \mathrm{H}_{5} \mathrm{OH}$ and $\mathrm{N}_{2} \mathrm{O}$ the individual bursts of radiation have varying amplitude and frequency interleaving, which depend on the condensation pressure. We analyzed transient behavior of individual pulses with a frequency of $10^{5}$ measurements per second. Figure 2 illustrates these results

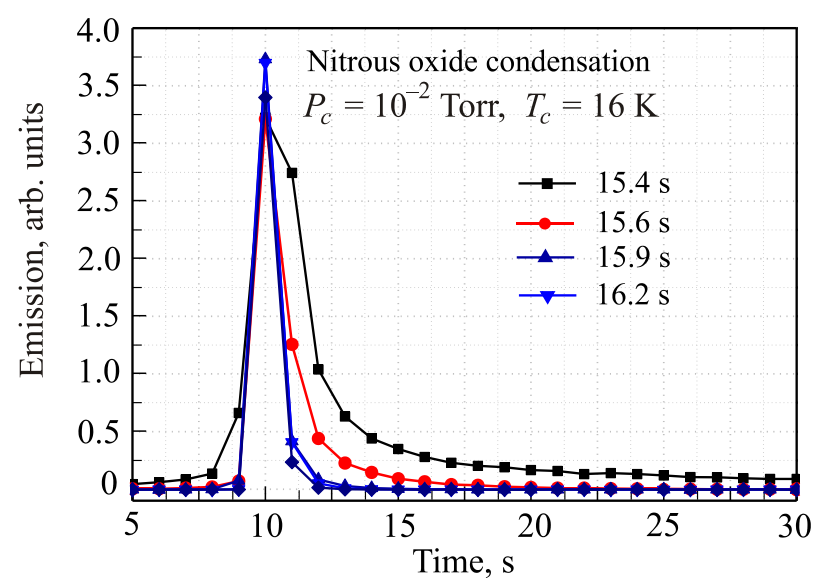

Fig. 2. (Color online) Time dependencies of individual flashes of cryocondensation emission in nitrous oxide. Frequency of data points is $10^{5}$ measurements per second. The condensation temperature is $T_{c}=16 \mathrm{~K}$. The pressure of the gas phase during condensation is $P_{c}=10^{-2}$ Torr. for nitrous oxide at different time intervals during condensation at $T_{c}=16 \mathrm{~K}$ and $P_{c}=10^{-2}$ Torr. In those transient plots, the countdown begins $5 \cdot 10^{-5} \mathrm{~s}$ before the maximum intensity of the radiation is reached.

One can observe the following important circumstance. Although the measurements were performed at a frequency of $10^{5}$ data points per second, they practically failed to register time-dependence of the rising edge of each individual flash, i.e., the duration of the onsets luminescence bursts is clearly less than $10^{-5} \mathrm{~s}$. The figure also shows that the relaxation time of the flash (the time in which the signal decreases from the maximum value to zero) is of the order of $6 \cdot 10^{-5}-10^{-4} \mathrm{~s}$.

Figure 3 shows the data for the transient behavior of individual pulses of cryocondensation radiation in ethanol. For the ease of comparison the results are normalized to have the same maximum values of the photomultiplier signal. The curves show that the dynamic nature of the flashes detected at different time intervals is essentially the same. The duration of onsets of radiation bursts is also shorter than $10^{-5} \mathrm{~s}$, similar to the case of nitrous oxide. However, the decrease of the signal occurs much slower than for the cryocondensation emission in nitrous oxide.

From Figs. 2 and 3 it may seem that cryoemission of nitrous oxide and ethanol is comprised of single (elementary) flashes. However, a more detailed analysis of the signals shown in these figures indicates that the individual pulses may represent a superposition of elementary flashes. This is clearly demonstrated in Fig. 4 showing the time distribution of the photomultiplier signal recorded during the condensation of nitrous oxide. It is noteworthy that the occurrence of the overlapping elementary pulses should be internally self-consistent for such observed cumulative time distribution of the radiation to occur. This result holds for the emission in ethanol as well.

Comparison of the relaxation times and the shapes of the flashes with similar amplitudes in nitrous oxide and

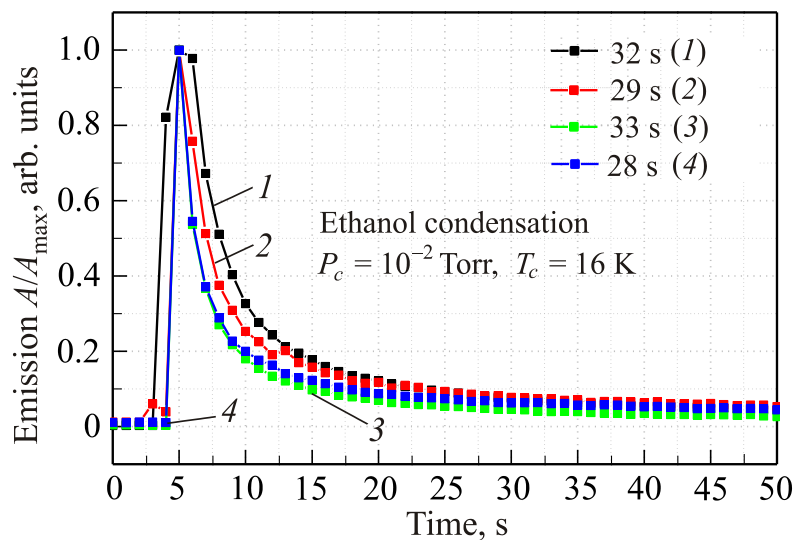

Fig. 3. (Color online) Time dependencies of individual flashes of cryocondensation emission in ethanol. Frequency of data points is $10^{5}$ measurements per second. The condensation temperature is $T_{c}=16 \mathrm{~K}$. The pressure of the gas phase is $P_{c}=10^{-2}$ Torr. 


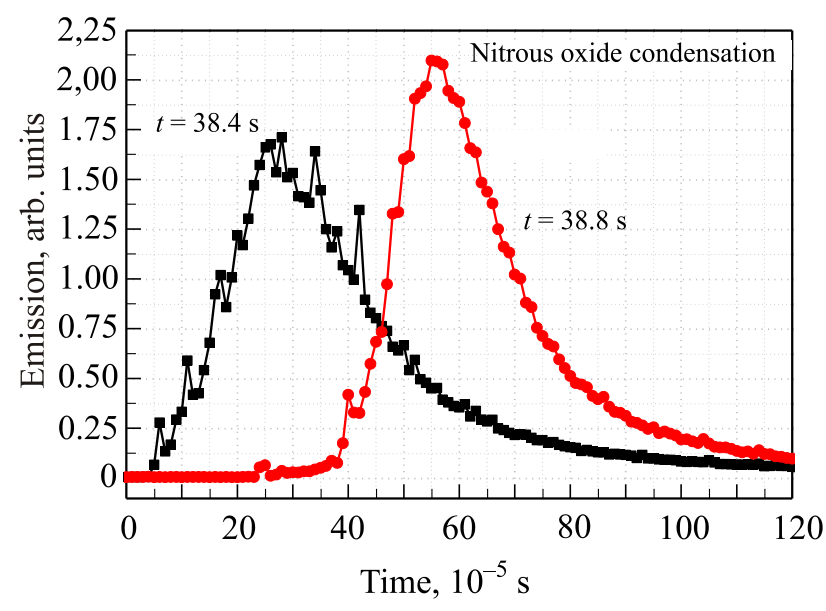

Fig. 4. (Color online) Photomultiplier signal representing a superposition of elementary pulses of the cryocondensation emission in nitrous oxide at two different time intervals.

ethanol is shown in Fig. 5. One can see significant differences, such as, e.g., the relaxation time of the radiation flash is much greater in ethanol compared to nitrous oxide and is of the order of $3 \cdot 10^{-4} \mathrm{~s}$.

It is clear that the analysis of the luminescence at a rate of $10^{5}$ samples per second is not adequate to resolve the time profiles of individual flashes, specifically such parameters as the onset times of reaching the maximum intensity and the relaxation times. Moreover, it is not clear whether the decrease of the photomultiplier signal reflects the proper relaxation process of an analyzed radiation pulse. To solve this problem, an experimental setup has been modified by an addition of an analog-to-digital converter allowing measurements of the dynamic characteristics of radiation at a rate of $10^{7}$ per second.

Results of these measurements are presented in Fig. 6, where one can see that the time to reach the maximum of the radiation flash is of the order of $7 \cdot 10^{-7} \mathrm{~s}$. According to our preliminary results, this time increases with the ampli-

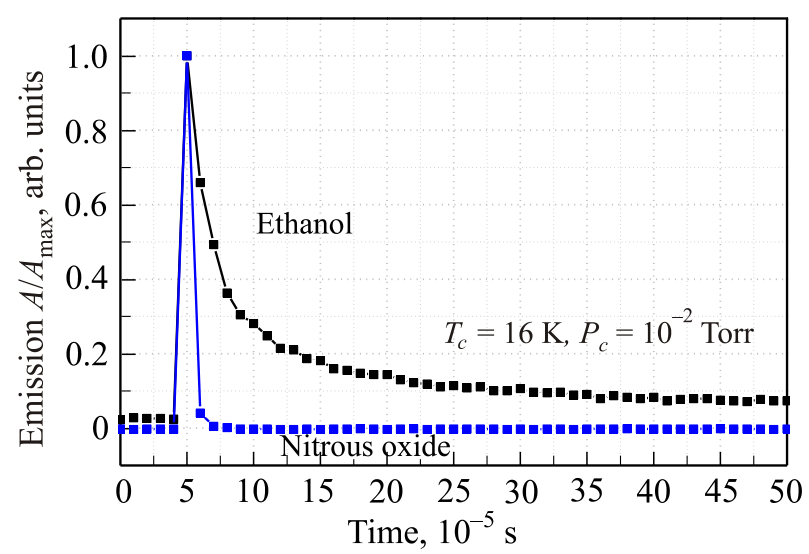

Fig. 5. (Color online) Comparison of the relaxation times and the shapes of the elementary cryoradiation bursts in nitrous oxide and ethanol.

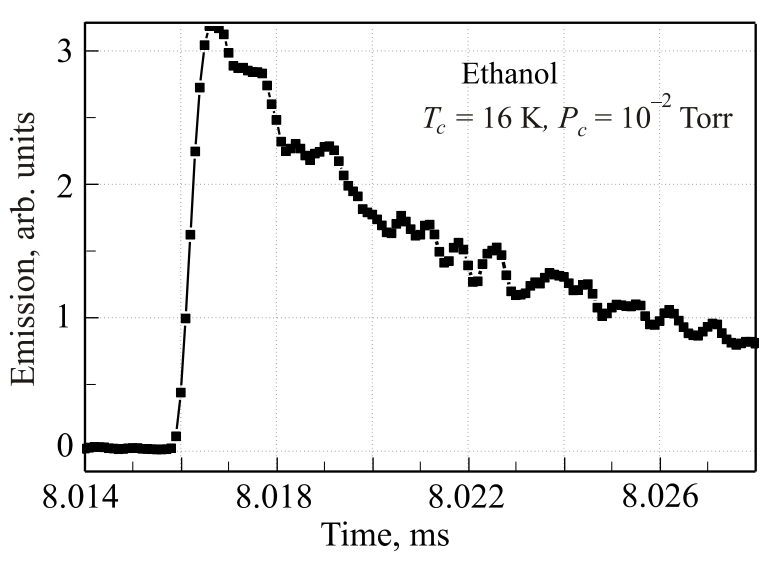

Fig. 6. Dynamic characteristics of a single flash of cryoradiation in ethanol measured with a frequency of $10^{7} \mathrm{~s}^{-1}$.

tude of the signal. Besides, it is noteworthy that what was first perceived as the process of relaxation of a single burst is, in principle, a superposition of many flashes. Interestingly, the intensity of these individual flashes varies smoothly over time, forming an envelope, which is perceived as a relaxation curve.

\section{Conclusions}

Based on the data presented above, we can conclude the following.

1. Of the compounds tested only nitrous oxide and ethanol exhibited the ability to generate cryoradiation during condensation from the gas phase. Existence or absence of an intrinsic dipole moment is not a necessary prerequisite for this cryoradiation to occur. Lack of cryocondensation radiation in carbon dioxide, water and argon may serve as an indirect confirmation of the assumption that the cryoemission itself of nitrous oxide and ethanol is not associated with the processes of triboluminescence or cracking of the samples.

2. Separate flashes detected by a photomultiplier represent a superposition of a series of successive relaxation processes of individual regions, somehow related to each other. It is possible that such areas are crystallites containing a set of molecules of nitrous oxide/ethanol, which are in metastable states with different activation energies. In such case, there could be certain energy distribution associated with the most probable metastable state for the majority of the molecules to be found in. Luminescence by these molecules during the transition into the ground state induces successive relaxation processes of molecular groups according to their activation energy distributions.

3 . Nonequilibrium radiative states forming in the process of cryocondensation of nitrous oxide and ethanol may have different nature of the activation energy distribution and this could explain the difference in the character of the radiation relaxation in these substances. 
1. D. Garypogly and A. Drobyshev, Fiz. Nizk. Temp. 16, 936 (1990) [Sov. J. Low Temp. Phys. 16, 547 (1990)].

2. A.S. Drobyshev and E.A. Samyshkin, Instrum. Exp. Techn. 40, 437 (1997).

3. A. Drobyshev, A. Aldiyarov, E. Korshikov, V. Kurnosov, D. Sokolov, and N. Tokmoldin, Fiz. Nizk. Temp. 39, 591 (2013) [Low Temp. Phys. 39, 460 (2013)].

4. R. Balog, P. Cicman, N.C. Jones, and D. Field, Phys. Rev. Lett. 102, 073003 (2009).

5. D. Field, O. Plekan, A. Cassidy, R. Balog, N.C. Jones, and J. Dunger, Int. Rev. Phys. Chem. 32, 345 (2013).

6. A. Cassidy, O. Plekan, R. Balog, N.C. Jones, and D. Field, Phys. Chem. Chem. Phys. 15, 108 (2013).

7. O. Plekan, A. Cassidy, R. Balog, N.C. Jones, and D. Field, Phys. Chem. Chem. Phys. 14, 9972 (2012).
8. A. Cassidy, O. Plekan, R. Balog, J. Dunger, D. Field, and N.C. Jones, J. Phys. Chem. A 118, 6615 (2014).

9. A. Cassidy, O. Plekan, J. Dunger, R. Balog, N.C. Jones, J. Lasne, A. Rosu-Finsen, M. McCoustra, and D. Field, Phys. Chem. Chem. Phys. 16, 23843 (2014).

10. G. Geballos, H. Wende, K. Baberschke, and D. Arvanitis, Surface Science 482-485, 15 (2001).

11. K.J. Franke, I. Fernandez-Torrente, J.I. Pascual, and N. Lorente, Phys. Chem. Chem. Phys. 10, 1640 (2008).

12. W.C. Trogler, Coord. Chem. Rev. 187, 303 (1999).

13. F. Wang and R.D. Harcourt, J. Phys. Chem. 104, 1304 (2000).

14. A. Drobyshev, A. Aldiyarov, E. Korshikov, D. Sokolov, and V. Kurnosov, Fiz. Nizk. Temp. 38, 1340 (2012) [Low Temp. Phys. 38, 1058 (2012)]. 